\title{
Generalized Aitken-like Acceleration of the Schwarz Method
}

\author{
Jacques Baranger ${ }^{1}$, Marc Garbey ${ }^{2}$, and Fabienne Oudin-Dardun ${ }^{1}$ \\ 1 University Lyon 1, Modelisation and Scientific Computing \\ 2 University of Houston, Computer Science (http://www.cs.uh.edu/ garbey/)
}

\begin{abstract}
Summary. (and Introduction) In this paper, we present a family of domain decomposition based on Aitken like acceleration of the Schwarz method seen as an iterative procedure with linear rate of convergence. This paper is a generalization of the method first introduced in Garbey and Tromeur-Dervout [2001] that was restricted to Cartesian grids. The general idea is to construct an approximation of the eigenvectors of the trace transfer operator associated to dominant eigenvalues and accelerate these components after few Schwarz iterates. We consider here examples with the finite volume approximation on general quadrangle meshes of Faille [1992] and finite element discretization.
\end{abstract}

\section{A General Framework for the Aitken-Schwarz Method}

Let us consider formally a linear differential problem

$$
L[U]=f \text { in } \Omega, U_{\mid \partial \Omega}=g .
$$

We assume that the problem is well posed and has a unique solution $U$. To simplify the presentation, we restrict ourselves to a domain decomposition of two overlapping subdomains $\Omega_{1} \cup \Omega_{2}=\Omega$, and we consider the additive version of the Schwarz algorithm (Smith et al. [1996]). We assume implicitly in the following notations that the Dirichlet boundary condition in (1) is satisfied by all intermediate subproblems. The Additive Schwarz (AdS) version of the algorithm writes,

$$
\begin{aligned}
& L\left[u_{1}^{n+1}\right]=f \text { in } \Omega_{1}, u_{1 \mid \Gamma_{1}}^{n+1}=u_{2 \mid \Gamma_{1}}^{n}, \\
& L\left[u_{2}^{n+1}\right]=f \text { in } \Omega_{2}, u_{2 \mid \Gamma_{2}}^{n+1}=u_{1 \mid \Gamma_{2}}^{n} .
\end{aligned}
$$

Because $L$ is linear, the following operator $T^{a}$ is linear:

$$
\left(u_{1 \mid \Gamma_{1}}^{n}-U_{\mid \Gamma_{1}}, u_{2 \mid \Gamma_{2}}^{n}-U_{\mid \Gamma_{2}}\right) \rightarrow\left(u_{1 \mid \Gamma_{1}}^{n+1}-U_{\mid \Gamma_{1}}, u_{2 \mid \Gamma_{2}}^{n+1}-U_{\mid \Gamma_{2}}\right) .
$$


Let us proceed with the discretized version of the problem (1), with solution $U^{h}$. For $i=1,2$, let $\Gamma_{i}^{h}$ be the set of mesh nodes corresponding to approximation of $U$ on $\Gamma_{i}, E_{i}^{h}$ a finite vector space used to approximate the solution restricted to the artificial interface $\Gamma_{i}^{h}$, and $\left\{b_{i}^{j}, j=1 \ldots N\right\}$ a set of basis functions for this vector space. We suppose that both vector space $E_{1}^{h}$ and $E_{2}^{h}$ have the same dimensions and define now the following two linear operators

$$
\begin{gathered}
T_{l}^{a}: u_{1 \mid \Gamma_{1}}^{n}-U_{\Gamma_{1}} \rightarrow u_{2 \mid \Gamma_{2}}^{n+1}-U_{\Gamma_{2}} \\
T_{r}^{a}: u_{2 \mid \Gamma_{2}}^{n}-U_{\Gamma_{2}} \rightarrow u_{1 \mid \Gamma_{1}}^{n+1}-U_{\Gamma_{1}} .
\end{gathered}
$$

Using the discrete representation of the interface $\Gamma_{i}^{h}$ in $E_{i}^{h}$, for $i=1,2$, we have

$$
\left(u_{2, j}^{n+1}-U_{j, \Gamma_{2}}\right)_{j=1, \ldots, N}=P_{l}\left(u_{1, j}^{n}-U_{j, \Gamma_{1}}\right)_{j=1, \ldots, N}
$$

and

$$
\left(u_{1, j}^{n+1}-U_{j, \Gamma_{1}}\right)_{j=1, \ldots, N}=P_{r}\left(u_{2, j}^{n}-U_{j, \Gamma_{2}}\right)_{j=1, \ldots, N},
$$

with $P_{l}$ (resp. $\left.P_{r}\right)$ square matrix of $T_{l}^{a}$ (resp. $T_{r}^{a}$ ). The matrix of the trace operator $T^{a}$ has then the characteristic anti diagonal structure

$$
P=\left(\begin{array}{cc}
0 & P_{r} \\
P_{l} & 0
\end{array}\right)
$$

The Additive Aitken Schwarz algorithm is then

- Step AdS0: compute $P_{l}$ and $P_{r}$.

- Step AdS1: from initial artificial interface condition $u_{1}^{0}$ and $u_{2}^{0}$ compute the first Schwarz iterate $(2,3)$.

- Step AdS2: from $u_{i}^{0}$ and $u_{i}^{1}(i=1,2)$ and the linear system $(7,8)$, get the exact interface value $U_{j, \Gamma_{1}}$ and $U_{j, \Gamma_{2}}$.

- Step AdS3: starting from the interface condition $U_{\mid \Gamma_{1}}=\sum_{j=1 . . N} U_{j, \Gamma_{1}} b_{1}^{j}$ and $U_{\mid \Gamma_{2}}=\sum_{j=1 . . N} U_{j, \Gamma_{2}} b_{2}^{j}$, apply one last Schwarz iterate $(2,3)$ to get $U^{h}$.

Iff $\left\|P_{l} P_{r}\right\|<1$, the additive Schwarz algorithm converges and the matrix $P$ associated to $(7,8)$ is non singular. This Aitken-Schwarz algorithm is then an exact solver.

Step AdS0 is the critical step of this algorithm; a straightforward and very expansive way to obtain $P$ consist in computing before hand in parallel the solution of $2 N$ independent sequences of homogeneous problem; alternatively, one may reconstruct these matrices using $2(N+1)$ consecutive iterates of the Schwarz method, but existence of the solution and stability of the numerical process is not guaranteed(Garbey and Tromeur-Dervout [2002]).

To find a numerically efficient method to compute $P$ or an approximation of $P$ is the key problem that we will address in the next sections. We are going to simplify the problem and show that our algorithm can be formulated with an approximation of the eigenvectors of the trace transfer operator that has the dominant eigenvalues. 


\section{Quasi-Diagonal Aitken-Schwarz Procedure}

Let us assume that $P_{l}$ (resp. $P_{r}$ ) can be diagonalized in the basis of eigenvectors $V_{j}$ corresponding to eigenvalues $\Lambda_{j}^{l}$ (resp. eigenvectors $W_{j}$ corresponding to eigenvalues $\Lambda_{j}^{r}$.)

Let us denote by $\left(\tilde{u}_{i, j}^{n}\right)_{j=1, \ldots, N}\left(\operatorname{resp} .\left(\hat{u}_{i, j}^{n}\right)_{j=1, \ldots, N}\right)(i=1,2)$ the components of $u_{i \mid \Gamma_{i}}^{n}$ in basis $\left\{V_{j}, j=1, \ldots, N\right\}$ (resp. $\left.\left\{W_{j}, j=1, \ldots, N\right\}\right)$.

Then, we have

$$
\left(\tilde{u}_{2, j}^{n+1}-\tilde{U}_{j \mid \Gamma_{2}}\right)_{j=1, . ., N}=D^{l}\left(\tilde{u}_{1, j}^{n}-\tilde{U}_{j \mid \Gamma_{1}}\right)_{j=1, . ., N},
$$

with $D_{j, j}^{l}=\lambda_{j}^{l}$, and in the basis of eigenvectors $W_{j}$,

$$
\left(\hat{u}_{1, j}^{n+1}-\hat{U}_{j \mid \Gamma_{1}}\right)_{j=1, . ., N}=D^{r}\left(\hat{u}_{2, j}^{n}-\hat{U}_{j \mid \Gamma_{2}}\right)_{j=1, . ., N}
$$

with $D_{j, j}^{r}=\lambda_{j}^{r}, j=1 . . N$. In order to compute $U_{j \mid \Gamma_{i}}, i=1 . .2$, we express both identities (9) and (10) in the same basis. We obtain in vector notations $u=\left(u_{j}\right)_{j=1 . . N}$, in $b_{j}^{i}, i=1,2$ basis,

$$
\begin{aligned}
U_{\Gamma_{1}}-\Lambda^{r} U_{\Gamma_{2}} & =u_{1}^{1}-\Lambda^{r} u_{2}^{0}, \\
-\Lambda^{l} U_{\Gamma_{1}}+U_{\Gamma_{2}} & =u_{2}^{1}-\Lambda^{l} u_{1}^{0},
\end{aligned}
$$

where $\Lambda^{l}=V D^{l} V^{-1}$, and $\Lambda^{r}=W D^{r} W^{-1}$.

The Quasi Diagonal Additive Schwarz algorithm writes

- Step QD-AdS0: compute approximate main eigenvectors $\left(\hat{V}_{j}\right)_{j=1 . . q}(\operatorname{resp}$. $\left.\left(\hat{W}_{j}\right)_{j=1 . . q}\right)$ and corresponding approximate eigenvalues $\left(\hat{\Lambda}_{j}^{l}\right)_{j=1 . . q}(\operatorname{resp}$. $\left.\left(\hat{\Lambda}_{j}^{r}\right)_{j=1 . . q}\right)$ of $P_{l}$ (resp. $\left.P_{r}\right)$.

- Step QD-AdS1: from initial artificial interface conditions $u_{1}^{0}$ and $u_{2}^{0}$, compute the first Schwarz iterate $(2,3)$.

- Step QD-AdS2: decompose $u_{1}^{0}$ and $u_{1}^{1}$ into the main components $u_{e, 1}^{0}$ and $u_{e, 1}^{1}$ (projection on $\left.\operatorname{span}\left[\hat{V}_{1}, \ldots, \hat{V}_{q}\right]\right)$ and the residuals $u_{r, 1}^{0 / 1}=u_{1}^{0 / 1}-\sum_{j=1 . . q} u_{e, j}^{0 / 1} \hat{V}_{j}$. Decompose $u_{2}^{0 / 1}$ in a similar way using the projection on $\operatorname{span}\left[\hat{W}_{1}, \ldots, \hat{W}_{q}\right]$

- Step QD-AdS3: from the formula (11) restricted to $\operatorname{span}\left[\hat{W}_{1}, \ldots, \hat{W}_{q}\right]$ and (12) restricted to $\operatorname{span}\left[\hat{V}_{1}, \ldots, \hat{V}_{q}\right]$ with corresponding approximated eigenvalues, get the (approximated) interface value $\hat{U}_{e, 1 / 2, j}, \forall j=1 . . q$.

- Step QD-AdS4: recompose the interface conditions from the following approximations $(i=1,2)$

$$
U_{\mid \Gamma_{1}} \approx \hat{U}_{\mid \Gamma_{1}}=\sum_{j=1 . . q}\left(u_{1, e, j}^{1}-\hat{\Lambda}_{j}^{l} u_{1, e, j}^{0}\right) /\left(1-\hat{\Lambda}_{j}^{l}\right) \hat{V}_{j}+u_{r, 1}^{1},
$$

(similarly for $U_{\mid \Gamma_{2}}$, with $\hat{\Lambda}^{r}$ and $\operatorname{span}\left[\hat{W}_{1}, \ldots, \hat{W}_{q}\right]$ ), and apply one Schwarz iterate $(2,3)$ to get an approximation of $U^{h}$. 
The following theorem summarizes the impact of the error on eigenvectors, the error on eigenvalues and the truncation parameter $q$ on the approximation of the artificial interfaces obtained with the quasi-diagonal additive Aitken Schwarz algorithm.

Theorem 1. Let $\left\{V_{1}, \ldots, V_{q}\right\}$ (resp. $\left.\left\{W_{1}, \ldots, W_{q}\right\}\right)$ be a set of $q$ independent eigenvectors of the trace transfer operator $P_{l}$ (resp. $\left.P_{r}\right)$. We suppose $\left\|P_{l / r}\right\|=$ $O(1)$. Let $\left\{\hat{V}_{1}, \ldots, \hat{V}_{q}\right\}$ (resp. $\left\{\hat{W}_{1}, \ldots, \hat{W}_{q}\right\}$ ) be a set of $q$ independent vectors such that the matrix $\epsilon_{l}$ (resp. $\epsilon_{r}$ ) of column vectors $\epsilon_{l, j}=\hat{V}_{j}-V_{j}$ (resp. $\left.\epsilon_{r, j}=\hat{W}_{j}-W_{j}\right)$, has norm $\left\|\epsilon_{l / r}\right\|=o(1)$.

Let us assume that $\delta_{j}^{l / r}=\left|\hat{\lambda}_{j}^{l / r}-\lambda_{j}^{l / r}\right|=o(1), \forall j=1 \ldots q$, and that $\operatorname{dist}\left(u_{1}^{0}-\right.$ $\left.U_{\mid \Gamma_{1}}, \operatorname{span}\left[\hat{V}_{1}, \ldots, \hat{V}_{q}\right]\right)+\operatorname{dist}\left(u_{2}^{0}-U_{\mid \Gamma_{2}}, \operatorname{span}\left[\hat{W}_{1}, \ldots, \hat{W}_{q}\right]\right)=\mu$, with $\mu=o(1)$, then

$$
\begin{array}{r}
\left\|\left(\hat{U}_{\mid \Gamma_{1}}-U_{\mid \Gamma_{1}}, \hat{U}_{\mid \Gamma_{2}}-U_{\mid \Gamma_{2}}\right)\right\|= \\
C^{t}\left\|\left(I d-\Lambda^{l} \Lambda^{r}\right)^{-1}\right\| O(\|\epsilon\|)+O(\|\beta\|)+O(\mu),
\end{array}
$$

with $\beta$ 2q-vector of components $\left(\beta_{j}^{l}=\frac{\delta_{j}^{l}}{\left|1-\lambda_{j}^{l}\right|}, \beta_{j}^{r}=\frac{\delta_{j}^{r}}{\left|1-\lambda_{j}^{r}\right|}\right)$.

Proof. See Garbey [2003]

This theorem suggests to get an approximation of the eigenvectors of the matrices $P_{l}, P_{r}$ corresponding to the dominant eigenvectors that is numerically cheap to compute. The Quasi-diagonal Aitken acceleration plays the role of a coarse grid preconditioner and can be iterated until convergence. We introduce in the following two examples of this construction, with respectively finite element discretization and then finite volume approximation.

\section{Finite Element on Tensorial Product of Two-D Grid}

We consider the homogenous Dirichlet boundary value problem (1) that has a separable second order operator $L=L_{1}+L_{2}$ :

$$
L_{1}=-\partial_{x}\left(a_{1} \partial_{x}\right)+b_{1} \partial_{x}+c_{1}, \quad L_{2}=-\partial_{y}\left(a_{2} \partial_{y}\right)+b_{2} \partial_{y}+c_{2}
$$

$a_{1}, b_{1}, c_{1}$ are functions of $\mathrm{x}$, and $a_{2}, b_{2}, c_{2}$ are functions of $\mathrm{y} . \Omega$ is a rectangle with a strip domain decomposition into rectangles. Interfaces of the domain decomposition are therefore parallel to the $y$ direction. The number of subdomains is arbitrary.

Let us consider the semi-discretisation of the operator in $y$ variable, with an irregular mesh in y $\left(y_{i}, i=0, \ldots, N+1\right), L_{2}^{k}$ a discretization of $L_{2}$ on the y-mesh, and $u^{i}(x)$ (resp. $\left.f^{i}(x)\right)$ an expected approximation of $u\left(x, y_{i}\right)$ (resp. $\left.f\left(x, y_{i}\right)\right)$. The semi-discrete approximation of a subdomain problem analogous to problem (2) or (3) is solved on a rectangle denoted by $\mathrm{R}=[\mathrm{e}, \mathrm{w}] \mathrm{x}[\mathrm{n}, \mathrm{s}]$ in order to simplify the notations: 


$$
\begin{array}{r}
\left.L_{1} u^{i}(x)+L_{2}^{k} u^{i}(x)=f^{i}(x), \quad x \in\right] e, w[ \\
u^{i}(w) \text { and } u^{i}(e) \text { given, } \quad u^{0}(x)=u^{N+1}(x)=0 .
\end{array}
$$

We introduce the eigenvalue problem:

$$
L_{2}^{k} \Phi_{j}=\lambda_{j} \Phi_{j}, \Phi_{j}^{0}=\Phi_{j}^{N+1}=0 .
$$

We set the hat transform :

$$
u^{i}(x)=\sum_{j=1}^{N} \hat{u}^{j}(x) \Phi_{j}^{i} ; i=1, \cdots, N
$$

with a similar expansion for $f^{i}(x)$. Applying this hat transform to (15-16) gives formally:

$$
\left.\sum_{j=1}^{N} \Phi_{j}^{i}\left[\left(L_{1}+\lambda_{j}\right) \hat{u}_{j}(x)-\hat{f}_{j}(x)\right)\right]=0, \quad \sum_{j=1}^{N} \Phi_{j}^{i} \hat{u}_{j}(e / w) \text { given }
$$

Following the notation of Theorem 1, the eigenvectors functions $V_{j}$ and $W_{j}$ of the trace transfer operator are identical and equal to the $\Phi_{j}, j=1 \cdots N$, functions. More precisely we have the following result for an arbitrary number of $P$ subdomains,

Theorem 2. Assume problem (17) has $N$ linearly independent real eigenvectors associated to real eigenvalues. Then each semi-discrete approximation of each subdomain problem is constituted of $N$ uncoupled continuous one dimensional linear problems $j=1, \cdots, N$ :

$$
\left[L_{1}+\lambda_{j}\right] \hat{u}_{j}(x)=\hat{f}_{j}(x), \quad \hat{u}_{j}(e / w) \text { given }
$$

The hat trace transfer operator is affine on $\mathbb{R}^{2 N(P-1)}$ with a block-diagonal matrix of $N$ blocks.

Proof. See Baranger et al. [2003]

From Theorem 1 one can estimate the number of eigenvectors $q$ that is worth to compute.

Problem (15-16) is closely related to finite difference point of vue. We now show that similar results can be obtained from the variational formulation of the problem.

Let us consider a semi discrete finite element approximation of the variational problem associated to (14). On a y-mesh we have a finite element space with basis function $\varphi_{m}, m=1, \cdots, N$. The unknown function is $u^{k}(x, y)=\sum_{m=1}^{N} u_{m}(x) \varphi_{m}(y)$ with $u_{m}(w / e)=0$. We obtain then the semi discrete variational problem: 


$$
\begin{aligned}
& \sum_{m} \int_{w}^{e}\left[a_{1} \partial_{x} u_{m} \partial_{x} v+\cdots\right] d x \int_{s}^{n} \varphi_{m} \varphi_{j} d y \ldots \\
& +\sum_{m} \int_{w}^{e} u_{m} v d x \int_{s}^{n}\left[a_{2} \partial_{y} \varphi_{m} \partial_{y} \varphi_{j}+\cdots\right] d y=\int_{R} f v \varphi_{j} d x d y
\end{aligned}
$$

Then the semi discrete variational problem (20) is: for all $m=1, \cdots, N$ find $u_{m}$ in $H_{0}^{1}(w, e)$ such that for all $v$ in $H_{0}^{1}(w, e)$ and $i=1, \cdots, N$

$$
\sum_{m}\left[\beta_{i m} \alpha^{1}\left(u_{m}, v\right)+\alpha_{i m} \beta^{1}\left(u_{m}, v\right)\right]=\beta^{1}\left(f_{i}, v\right)
$$

with

$$
\begin{gathered}
\alpha^{1}(u, v)=\int_{w}^{e}\left(a_{1} u_{x} v_{x}+b_{1} u_{x} v+c_{1} u v\right) d x, \alpha^{2}(u, v)=\int_{s}^{n}\left(a_{2} u_{y} v_{y}+b_{2} u_{y} v+c_{2} u v\right) d y \\
f_{i}(x)=\int_{s}^{n} f \varphi_{i} d y, \beta^{1}(u, v)=\int_{w}^{e} u v d x, \beta^{2}(u, v)=\int_{s}^{n} u v d y \\
\gamma_{i m}=\gamma^{2}\left(\varphi_{i}, \varphi_{m}\right)(\gamma=\alpha, \beta) .
\end{gathered}
$$

Using the generalized Fourier transform (18) we obtain from equation (21):

$$
\sum_{m} \sum_{j}\left[\beta_{i m} \alpha^{1}\left(\hat{u}_{j}, v\right)+\alpha_{i m} \beta^{1}\left(\hat{u}_{j}, v\right)-\beta_{i m} \beta^{1}\left(\tilde{f}_{j}, v\right)\right] \Phi_{j m}=0
$$

Choosing the $\Phi$ 's as the eigenvectors of the spectral problem:

$$
\sum_{m} \alpha_{i m} \Phi_{j m}=\lambda_{j} \sum_{m} \beta_{i m} \Phi_{j m}, j=1, \cdots, N
$$

gives to equation (22) the uncoupled form:

$$
\alpha^{1}\left(\hat{u}_{j}, v\right)+\lambda_{j} \beta^{1}\left(\hat{u}_{j}, v\right)=\beta^{1}\left(\tilde{f}_{j}, v\right) .
$$

We obtain then a result analogous to Theorem 2 . We are going now to show a second variant of this construction for finite volume approximation with general quadrangle meshes.

\section{An Example with Finite Volume on General Quadrangle Meshes}

We consider an approximation of (1) with the finite volume approximation on general quadrangle meshes of Faille [1992]. For simplicity we restrict ourselves to the Poisson operator with homogeneous Dirichlet boundary conditions. We also consider the multiplicative version of the Schwarz (MuS) algorithm with two subdomains. Those restrictions are not necessary, but they make the understanding of the construction easier. For general quadrangle meshes, an 
eigenvector basis of the trace transfer operator $T^{h}$ on the interface $u_{h \mid \Gamma^{h}}^{n} \rightarrow$ $u_{h \mid \Gamma^{h}}^{n+1}$, cannot be constructed analytically as in the previous section. We recall that

$$
u_{h \mid \Gamma^{h}}^{n}-U_{\mid \Gamma^{h}} \rightarrow u_{h \mid \Gamma^{h}}^{n+1}-U_{\mid \Gamma^{h}}
$$

is a linear operator and we denote by $P^{h}$ its matrix. $E^{h}$ is the finite vector space used to approximate the solution restricted to the interface $\Gamma^{h}$.

To represent the functions of $E^{h}$ in a more compact way, we choose the space of approximation $\mathcal{E}_{1}^{q}=\operatorname{span}[\sin (\tau), \sin (2 \tau), \ldots, \sin (q \tau)]$ where $\tau \in$ $(0, \pi)$ is a natural parameterization of the interface $\Gamma$. We introduce then the operator $\mathcal{T}_{h / q}$ from $E_{1}^{h, 0}$ to $\mathcal{E}_{1}^{q}$ that gives the least square approximation of grid function of $\Gamma^{h}$ with q-sine expansion:

$$
\sum_{k=1 . . q} a_{j}^{k} \sin (k \tau)
$$

Vice versa the operator $\mathcal{T}_{q / h}$ collocates the sinus expansions of $\mathcal{E}_{1}^{q}$ at grid points of $\Gamma^{h}$. The operator $T_{h / q} P^{h} T_{q / h}$ is linear. Let us denote by $P^{q}$ its matrix.

If the trace of the sub-domain solution at the artificial interface is regular enough, its approximation in $\mathcal{E}^{q}$ will have a much lower dimension than an approximation in $E^{h}$ for the same level of accuracy (Gottlieb and Shu [1997]). Furthermore for the approximation of the Poisson problem or the Helmholtz operator, in deformed rectangle, the sinus basis is somehow a natural basis.

We compute therefore directly the matrix $P^{q}$ with $q$ much smaller than the number of grid points. The column of $P^{q}$ are obtained by processing $q$ independent Schwarz iterates starting from $\sin (k \tau)$ for the artificial boundary condition. We choose the set of basis function $V_{j}=W_{j}, j=1 \ldots q$ to be the eigenvectors of $P^{q}$.

Figure 1 gives the convergence history of our method for a Poisson solver discretized with the Finite Volume method of Faille [1992] in a complex shape domain. The continuous line is the convergence history of MuS with no acceleration. The curve 'o' (resp. '+', '*') is for 2 waves (resp. 4, 8). We see that the convergence improves as the number of modes increases.

The trigonometric representation of the interface may not be the best solution in the general case, and there are many piecewise polynomial spaces of functions that might be more appropriate depending on the space of approximation of the PDE solution. This should be the topic of further investigations.

\section{Conclusion}

We have shown how to generalized the Aitken-Schwarz method from Cartesian grid with finite differences to other discretization such as finite element on tensorial product of grid or finite volumes on general quadrangle meshes. Let us emphasis that the implementation of our method can reuse the initial 


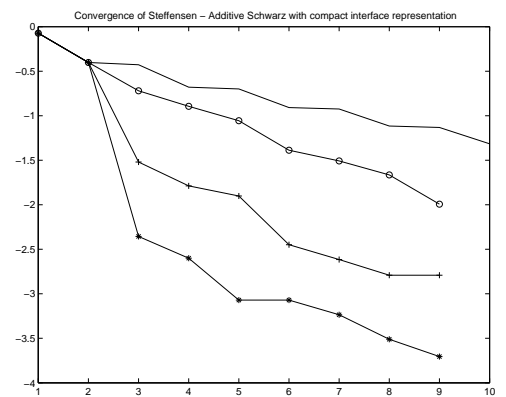

Fig. 1. Central Finite Volume discretisation: compact grid interface, overlap is 5 per cent, grid is $81 \times 81$.

coding of the Schwarz method with no change. As a matter of fact, the computation of dominant eigenvectors of the trace transfer operator can be seen as a pre-processing step. This step may involve few independent parallel executions of one Schwarz iteration with the original code. Further the Aitken-like acceleration procedure itself operates on the trace generated by the Schwarz code and does not require any change in the data structure of the original code. As shown in Garbey and Tromeur-Dervout [2002] this approach gives efficient parallel implementation with slow network. This is the philosophy of our ongoing work on metacomputing of elliptic problems.

Acknowledgement. Research of the second author reported here was supported by Award 0305405 from the National Science Foundation.

References

J. Baranger, M. Garbey, and F. Oudin-Dardun. Acceleration of the Schwarz method : the cartesian grid with irregular space step case. Technical report, CDCSP (Center for the development of parallel scientific computing), 2003.

I. Faille. A control volume method to solve an elliptic equation in twodimensional irregular meshing. Comp. Meth. Appl. Mech. Engrg., 100:275290, 1992.

M. Garbey. Acceleration of the Schwarz method for elliptic problems. submitted, 2003.

M. Garbey and D. Tromeur-Dervout. Two level domain decomposition for multi-clusters. In T. Chan, T. Kako, H. Kawarada, and O. Pironneau, editors, 12th Int. Conf. on Domain Decomposition Methods, pages 325-339. DDM.org, 2001.

M. Garbey and D. Tromeur-Dervout. On some Aitken like acceleration of the Schwarz method. Int. J. for Numerical Methods in Fluids, 40 (12): 1493-1513, 2002.

D. Gottlieb and W. Shu, C. On the Gibbs phenomenom and its resolution. SIAM Review, 39(4):644-668, 1997.

B. F. Smith, P. E. Bjørstad, and W. Gropp. Domain Decomposition: Parallel Multilevel Methods for Elliptic Partial Differential Equations. Cambridge University Press, 1996. 